\title{
Psychophysiological predictors of effective adaptation to the allostatic load of the mountain climbers
}

\author{
S. Krivoschekov ${ }^{1 *}$, E. Nikolaeva ${ }^{2}$, E. Vergunov $^{1}$, N. Balioz ${ }^{1}$, Yu. Bobrova $^{3}$ \\ ${ }^{1}$ Scientific Research Institute of Physiology and Basic Medicine, Novosibirsk, Russia \\ ${ }^{2}$ Herzen State Pedagogical University, Saint-Petersburg, Russia \\ ${ }^{3}$ Novosibirsk State University of Economics and Management, Novosibirsk, Russia \\ *e-mail: krivosch@physiol.ru
}

Key words: mountain climbers, allostasis, go/no-go, hypoxia, heart rhythm, handedness, orthostasis

Motivation and Aim: A study of phenotypic characteristics of human adaptation to extreme factors of the external environment (such as, for example, hypoxia) represents the actual task and allows identifying the predictors of the organism's successful functioning [1]. It was found that the result of adaptation depends not only on the functional abilities of the organism and allostatic changes [2], but also on the volitional and personal characteristics of the subjects. Of particular interest is the study of human cognitive functions in the course of such adaptations [3]. It is shown that due to strongwilled qualities people can overcome physiological ailments [4] or to carry out excessive physical loading, in particular, at high-mountain ascents. The authors investigated the handedness, the reserves of the cardiovascular system and the features of sensorimotor integration in mountain climbers as possible predictors of adaptation to extreme factors of the external environment.

Methods and Algorithms: At qualified mountain climbers taking into account proficiency and age difficult sensorimotor reaction (go/no-go at normoxia and normobaric hypoxia) was studied, assessment of handedness and indicators of orthostatic test (A. Riftin's technique) were carried out.

Results: Stability of sensorimotor integration in climbers under hypoxia is higher than in normoxia. The correlation between the results of the shoulder test (handedness test) and the assessment of the cardiovascular system's reserves obtained on the basis of orthotest indicators (reserves higher for left-side preferences) is shown.

Conclusion: The relation of the regulation of the autonomic nervous system with lefthanders, resulting in more effective adaptation to the high altitude and the possibility of using handedness as a phenotypic predictor of the level of reserves of the climbers' cardiovascular system. It is suggested that the factor of hypoxia in climbers in certain conditions has a stimulating effect on the processes of sensorimotor integration.

Acknowledgements: Supported by the RFBR (18-013-00323, 17-06-00166).

\section{References}

1. Divert V.E. et al. (2017) Vegetative balance of the organism and chemoreactive properties of cardiorespiratory system at climbers. Siberian Scientific Medical Journal. 37(3):72-78.

2. Krivoschekov S.G. et al. (2016) The concept of allostasis in the connection with human adaptation in the North. Human Ecology. 7:17-25.

3. Vergunov E.G. et al. (2018) Lateral preferences as the possible phenotypic predictors of the reserves of the cardiovascular system and the features of sensorimotor integration in climbers. Human Physiology. 44(3).

4. Nikolaeva E.I. et al. (2016) The relationship between the quality of life, hardiness and parameters of autonomic balance. In: Behavior change: making an impact on health and health services. Aberdeen, Scotland: University of Aberdeen. 736-736. 